\title{
(2) OPEN ACCESS \\ Is extensive cardiopulmonary screening useful in athletes with previous asymptomatic or mild SARS-CoV-2 infection?
}

\author{
Salvatore Francesco Gervasi @ (0) ${ }^{1,2}$ Luca Pengue, ${ }^{1}$ Luca Damato, ${ }^{2}$ Riccardo Monti, ${ }^{2}$ \\ Silvia Pradella, ${ }^{3}$ Tommaso Pirronti, ${ }^{4}$ Alessandro Bartoloni, ${ }^{5}$ Francesco Epifani, ${ }^{6}$ \\ Alessio Saggese, ${ }^{6}$ Francesco Cuccaro, ${ }^{1}$ Massimiliano Bianco, ${ }^{1,2}$ Paolo Zeppilli, ${ }^{2}$ \\ Vincenzo Palmieri ${ }^{1,2}$
}

\begin{abstract}
${ }^{1}$ Sports Medicine Unit, Fondazione Policlinico Universitario Agostino Gemelli IRCCS, Rome, Italy

${ }^{2}$ Sports Medicine Unit, Catholic University of the Sacred Heart Faculty of Medicine and Surgery, Roma, Lazio, Italy

${ }^{3}$ Department of Emergency Radiology, University Hospital Careggi, Firenze, Toscana, Italy ${ }^{4}$ Diagnostic Imaging Area, Fondazione Policlinico Universitario Agostino Gemelli IRCCS, Rome, Italy ${ }^{5}$ Department of Experimental and Clinical Medicine, University Hospital Careggi, Firenze, Toscana, Italy

${ }^{6}$ Synlab Med, Sesto Fiorentino, Florence, Italy
\end{abstract}

\section{Correspondence to}

Dr Salvatore Francesco Gervasi, Catholic University of the Sacred Heart Faculty of Medicine and Surgery, Roma 00166, Lazio, Italy;

gervasi.salvatore.md@gmail.com

Accepted 23 September 2020 Published Online First

5 October 2020

\section{Check for updates}

(c) Author(s) (or their employer(s)) 2021. Re-use permitted under CC BY-NC. No commercial re-use. See rights and permissions. Published by BMJ.

To cite: Gervasi SF

Pengue L, Damato L,

et al. Br J Sports Med

2021:55:54-61

\section{ABSTRACT}

Objective During the COVID-19 pandemic, it is essential to understand if and how to screen SARSCoV-2-positive athletes to safely resume training and competitions. The aim of this study is to understand which investigations are useful in a screening protocol aimed at protecting health but also avoiding inappropriate examinations.

Methods We conducted a cohort study of a professional soccer team that is based on an extensive screening protocol for resuming training during the COVID-19 pandemic. It included personal history, antigen swabs, blood tests, spirometry, resting/stress-test ECG with oxygen saturation monitoring, echocardiogram, Holter and chest CT. We also compared the findings with prior data from the same subjects before infection and with data from SARS-CoV-2-negative players.

Results None of the players had positive swab and/ or anti-SARS-CoV-2 IgM class antibodies. Out of 30 players, $18(60 \%)$ had IgG class antibodies. None had suffered severe SARS-CoV-2-related disease, 12 (66.7\%) had complained of mild COVID-19-related symptoms and $6(33.3 \%)$ were asymptomatic. None of the players we examined revealed significant cardiovascular abnormalities after clinical recovery. A mild reduction in spirometry parameters versus pre-COVID-19 values was observed in all athletes, but it was statistically significant $(p<0.05)$ only in SARS-CoV-2-positive athletes. One SARS-CoV-2-positive player showed increased troponin I level, but extensive investigation did not show signs of myocardial damage.

Conclusion In this small cohort of athletes with previous asymptomatic/mild SARS-CoV-2 infection, a comprehensive screening protocol including blood tests, spirometry, resting ECG, stress-test ECG with oxygen saturation monitoring and echocardiogram did not identify relevant anomalies. While larger studies are needed, extensive cardiorespiratory and haematological screening in athletes with asymptomatic/mild SARSCoV-2 infection appears unnecessary.

\section{INTRODUCTION}

Since it was discovered in late 2019, the new coronavirus named SARS-CoV-2 and its related clinical illness (COVID-19) has dramatically changed the health systems, economy and lifestyle all around the world. One of the measures adopted to contain the spread of the virus was suspending almost all sports leagues and tournaments. With the gradual return to the 'new normal', questions arose about how to safely resume training and competition. ${ }^{12}$ The main issues are how to prevent new infections and screen athletes to exclude cardiovascular, pulmonary and other SARS-CoV-2-related conditions that may pose a risk to the athlete's health. It is documented that SARS-CoV-2 can cause, in addition to acute respiratory syndrome, myocarditis, myocardial damage, acute coronary syndromes, arrhythmias and thromboembolic diseases, ${ }^{34}$ although published data refer to hospitalised patients with severe disease and often with comorbidities. It is not clear, however, whether previously healthy subjects with asymptomatic or mild SARS-CoV-2 infection will later develop cardiovascular or respiratory complications and how often. Before resumption of sports, it is essential that SARS-CoV-2-positive athletes are adequately screened to decide if, when and how to resume training and competitions after resolution of infection. In Italy, a country that has always been at the forefront in the prevention of sudden cardiac death related to sports, ${ }^{5-7} 8$ different screening protocols for resuming activity have been proposed for professional athletes with known SARS-CoV-2 infection. $^{12}$

We have recently carried out an extensive cardiorespiratory screening for a professional high-level soccer team where numerous players were SARSCoV-2-positive, either asymptomatic or with mild symptoms. We compared data from SARSCoV-2-positive players with data from the same subjects before infection and with data from SARSCoV-2-negative players. The aim of the present study was to understand which investigations are useful in a screening protocol aimed primarily at protecting health but also avoiding inappropriate examinations.

\section{METHODS}

The study was conducted on a cohort of male professional soccer players using the preparticipation screening protocol suggested by the Medical Commission of the Italian Soccer Federation (FIGC) for resuming training after an interruption linked to the SARS-CoV-2 pandemic. According to the protocol, all players were preliminary subjected to the following:

1. Personal history. 
2. Rhinopharyngeal swab for SARS-CoV-2 RNA with real-time PCR method.

3. Search for anti-SARS-CoV-2 antibodies of IgG class (qualitative and quantitative) and of $\operatorname{IgM}$ class (qualitative) in a venous blood sample by immunochromatography and chemiluminescence.

Rhinopharyngeal swab was performed with AllPlex-Seegene CE-IVD (Seegene, Seoul, Republic of Korea). The qualitative blood test for the presence of anti-SARS-CoV-2 antibodies was performed using GCCOV-402A Kit (Zhejiang Orient Gene Biotech, Huzhou, Zhejiang, China). The quantitative blood test for IgG class anti-COVID-19 antibodies was performed with m2000 SARS-CoV-2 assay (Abbott Laboratories, Illinois, USA).

Based on the results of the preliminary screening, players were divided into three groups according to the FIGC protocol:

A. Players who suffered severe SARS-CoV-2-related disease requiring hospitalisation (for pneumonia).

B. Players who suffered mild symptomatic SARS-CoV-2-related disease or remained fully asymptomatic despite infection.

C. Players without evidence of the virus and remained asymptomatic during the pandemic.

Since none of the team players had suffered from severe disease, to simplify we classified group 2 (mild/asymptomatic disease) as the COVID-19+ group and group 3 as the COVID-19- group.

If the swab and/or the search for IgM class antibodies were positive, the player was sent back home with the recommendation for self-isolation, daily communication of his clinical condition and to follow the indications of the public health authorities. A retest was planned after 14 days.

If both the swab and the search for IgM class antibodies were negative but IgG class antibodies were positive, the athlete was included in the COVID-19+ group and subjected to the following after at least 15 days from clinical resolution:

1. Physical examination.

2. Blood tests: complete blood count (CBC) with formula, alanine transaminase (ALT)/aspartate transaminase (AST), gamma-glutamyl transferase (GGT), lactic acid dehydrogenase (LDH), creatine phosphokinase (CPK), C reactive protein (CRP), D-dimer, high-sensitivity troponin I (TnI) after at least 48 hours without training, interleukin (IL)-6, prothrombin time (PT), activated partial thromboplastin time (aPTT), international normalised ratio (INR) and creatinine.

3. Spirometry.

4. Resting and stress-test ECG with a cycle ergometer and incremental protocol of 50 watts $/ 2 \mathrm{~min}$, plus non-invasive monitoring of oxygen saturation.

5. Echocardiogram (ECHO).

6. 24-hour Holter ECG monitoring, including one heavy training session.

7. Chest CT.

If both the swab and the search for IgM and $\operatorname{IgG}$ class antibodies were negative, the player was included in the COVID19- group and subjected to standard investigations included in routine Italian preparticipation screening for professional soccer players ${ }^{9}$ :

1. Personal history and physical examination.

2. Blood tests (CBC with formula, ALT, AST, GGT, LDH, CPK, CRP, ferritin and creatinine).

3. Spirometry.

4. ECHO.

5. Resting and stress-test ECG.

Spirometry was performed with MIR Spirolab (MIR-Medical International Research USA, New Berlin, Wisconsin, USA). Resting ECG was performed with ECG 100+ (Cardioline SpA,
Trento, Italy), while stress-test ECG with Edan SE-1515 DX12 (Edan, Shenzhen, People's Republic of China) and Sana Bike $120 \mathrm{~F}$ (ergosana int, Bitz, Germany). Holter monitoring was performed with Edan SE-2012 (Edan). ECHO was performed with Vivid T8 Pro (GE Medical Systems, Chicago, Illinois, USA).

The following parameters were analysed:

1. Clinical profile: (1) history of pulmonary disease, arterial hypertension, coronary artery disease, congenital or acquired cardiac diseases, and diabetes; (2) symptoms both at the time of the study and/or in the previous months potentially related to SARS-CoV-2 infection (fever $>37.5^{\circ} \mathrm{C}$, cough, dyspnoea, asthenia, ageusia, anosmia, diarrhoea and headache) or suggesting cardiovascular involvement: exertional dyspnoea, palpitations, chest pain, presyncope and syncope.

2. Blood test anomalies.

3. Respiratory parameters (spirometry): forced vital capacity, forced expiratory volume in the first second $\left(\mathrm{FEV}_{1}\right)$, peak expiratory flow volume (PEF), and maximum voluntary ventilation, absolute and as percentage of the theoretical values for age and body size.

4. Resting ECG: heart rate (HR), arrhythmias, conduction disturbances and ventricular repolarisation anomalies.

5. Stress-test ECG: maximum HR reached (absolute and as percentage of the theoretical maximum HR for age), resting/peak blood pressure, supraventricular and/or ventricular arrhythmias, and/or T wave/ST segment anomalies, and/or conduction disturbances. Oxygen saturation at rest, peak effort and at fourth minute of recovery was assessed only in COVID-19+ players.

6. ECHO: left ventricular size (end-diastolic/systolic diameter, volume and wall thicknesses), kinetics and systolic/diastolic function indexes (ejection fraction, mitral E:A ratio, tissue Doppler imaging at the mitral annulus); right ventricle size (RVD1, RVD2), kinetics and function (tricuspid annular plane systolic excursion); and presence of pericardial effusion.

7. Holter (only in COVID-19+ players): clinically relevant bradycardia/tachyarrhythmias and/or conduction disturbances.

8. Chest CT (only in COVID-19+ players): pulmonary and/or vascular parenchymal anomalies.

The results of the tests were then classified as 'normal' in the absence of anomalies in all parameters or 'abnormal' in case of anomalies in one or more parameters. The clinical profiles and the results of blood tests, spirometry, resting/stress-test ECG and ECHO were compared between (1) COVID-19+ and COVID19- groups; and (2) in the players of the COVID-19+ group, between the examinations carried out on the same athletes during the preseason of the previous year (9-11 months before) and after the outbreak.

Written consent was obtained from all subjects.

\section{Patient and public involvement}

Patients or the public were not involved in the design, conduct, reporting or dissemination plans of our research.

\section{Statistical analysis}

A descriptive analysis of all the sample parameters collected was carried out. Since we could not assume the normal distribution through the Shapiro-Wilk test, the variables were reported as median and IQR. The qualitative variables were presented as absolute frequencies and percentages (\%). Medians were compared between the groups by Mann-Whitney tests, and 


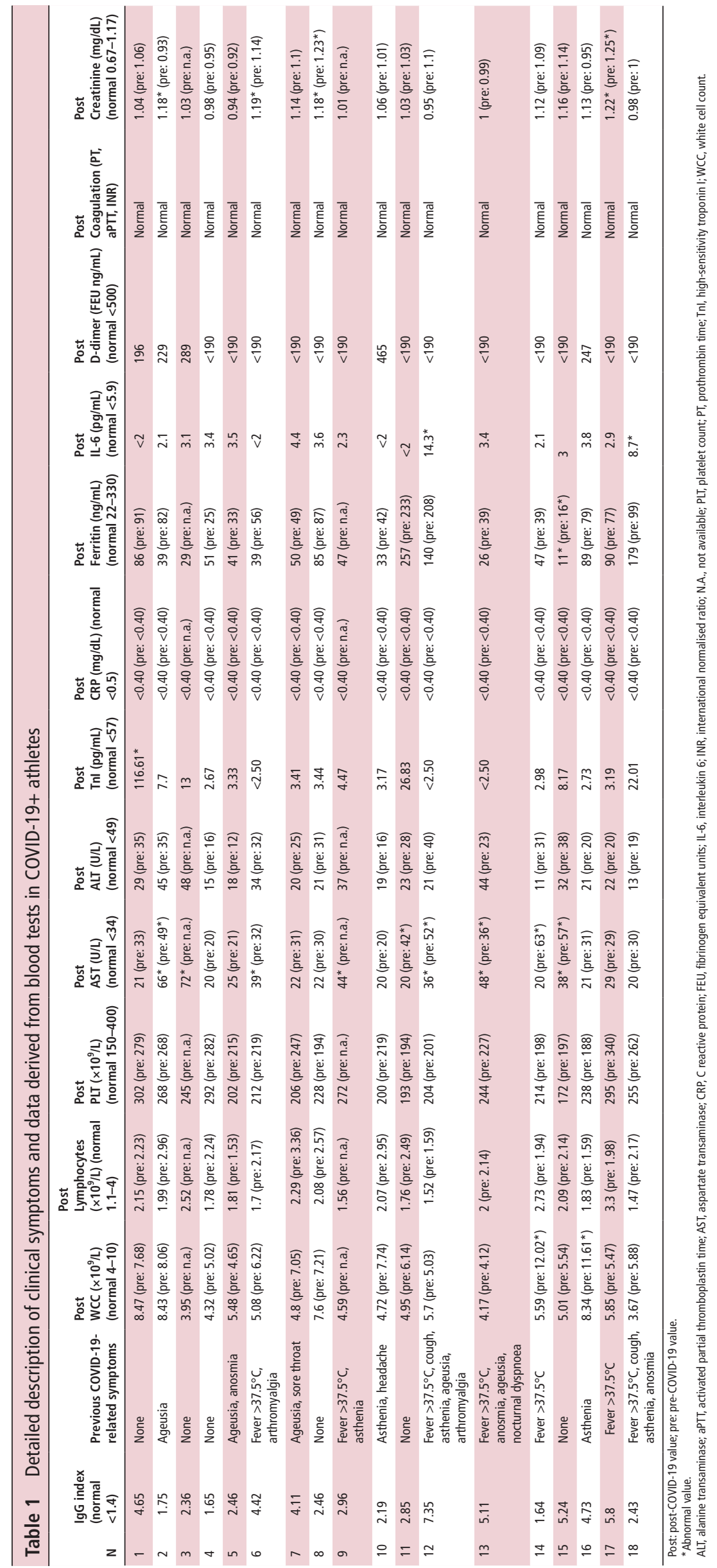




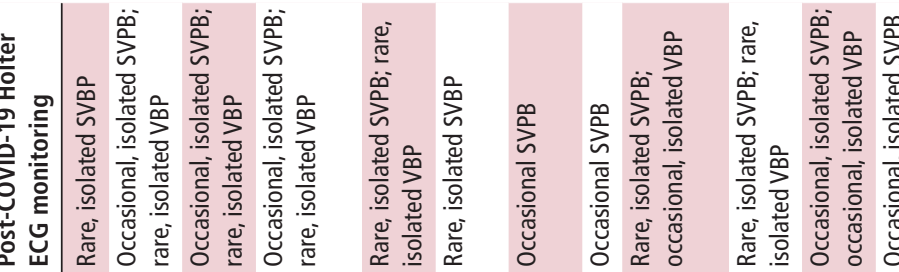

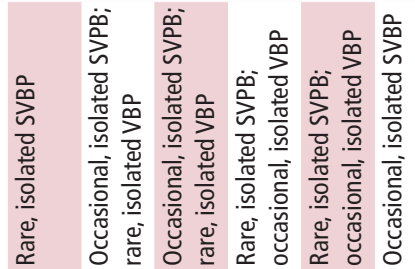

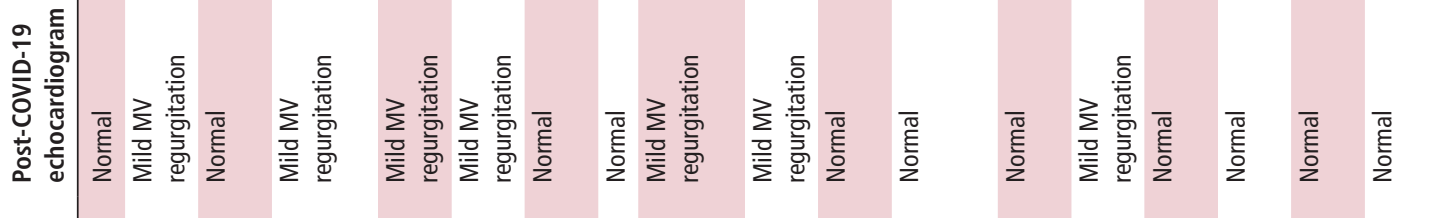

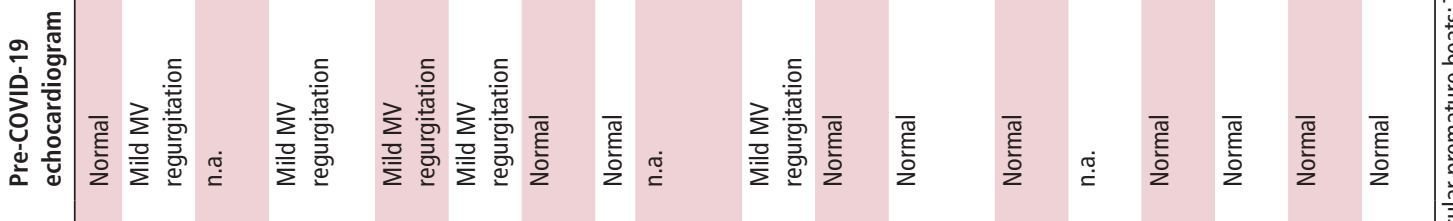

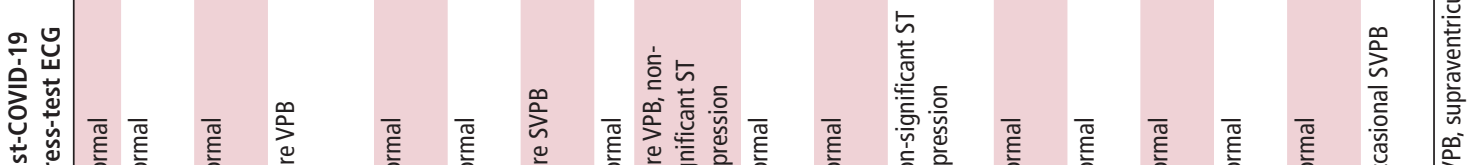

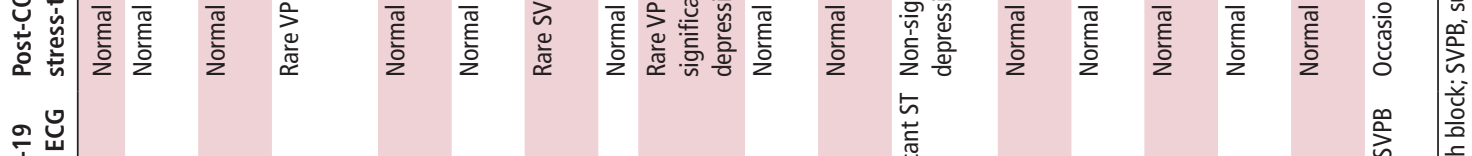

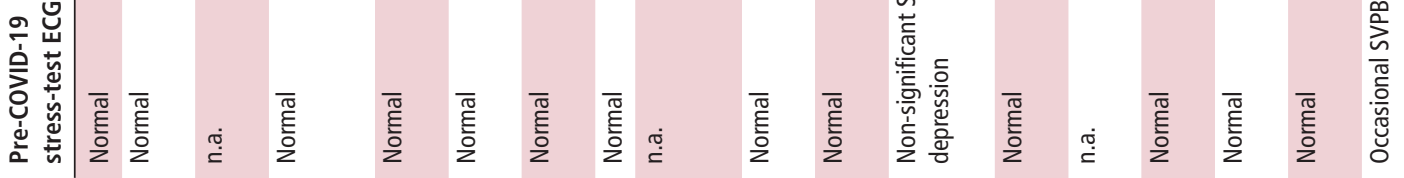

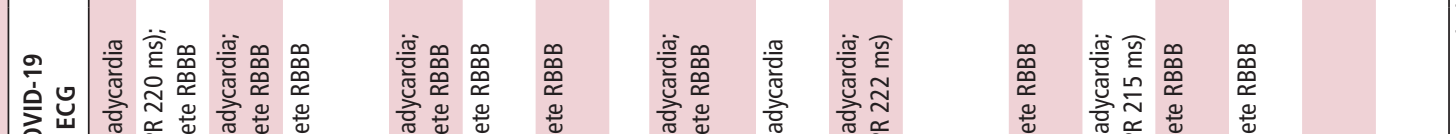

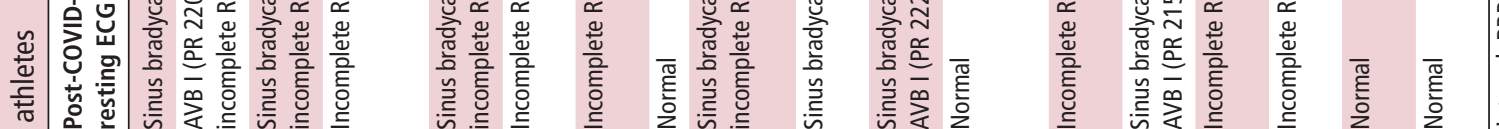

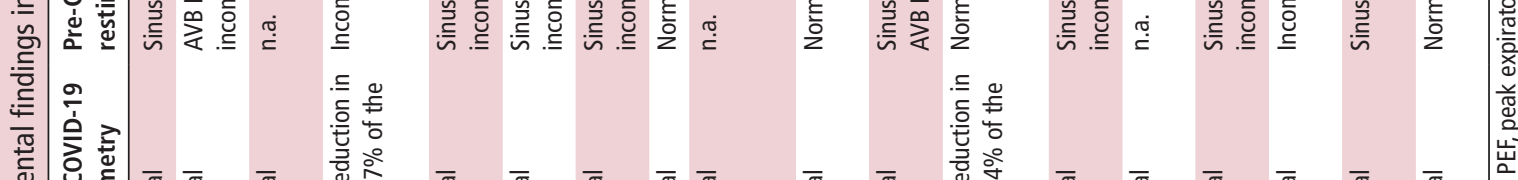

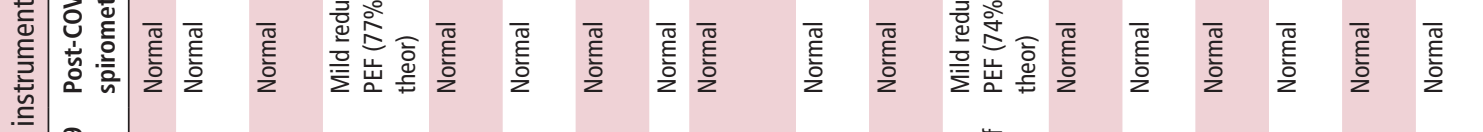

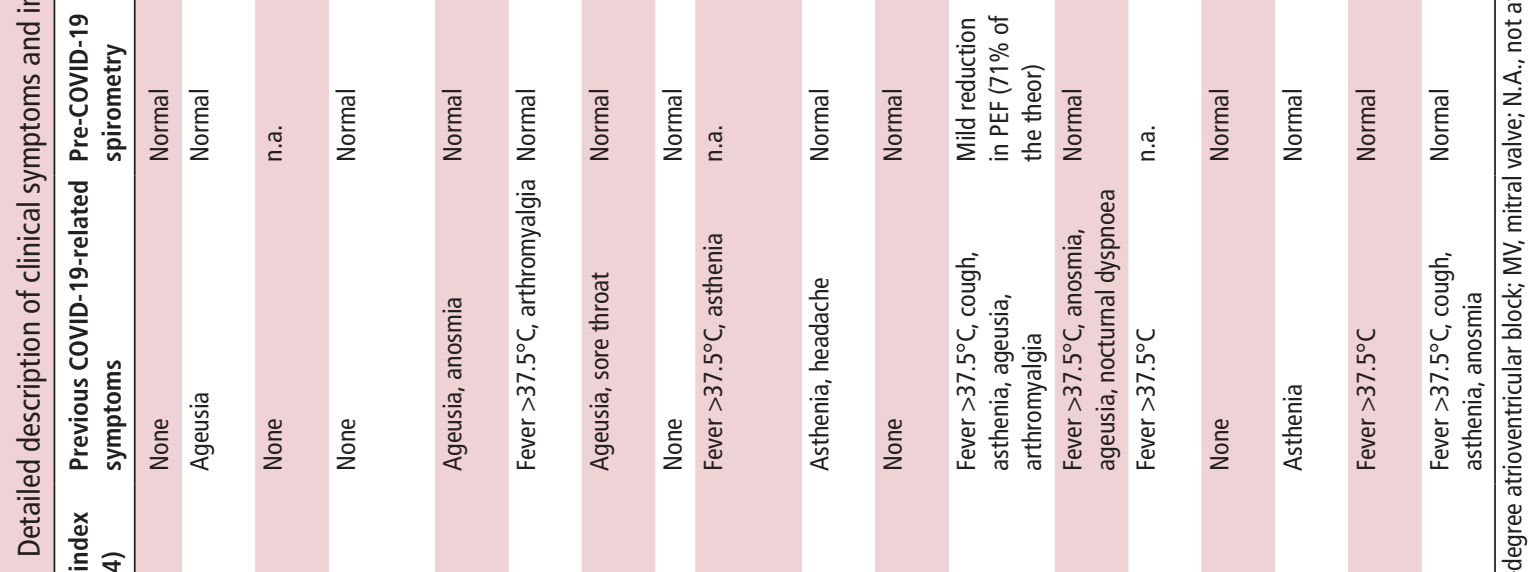

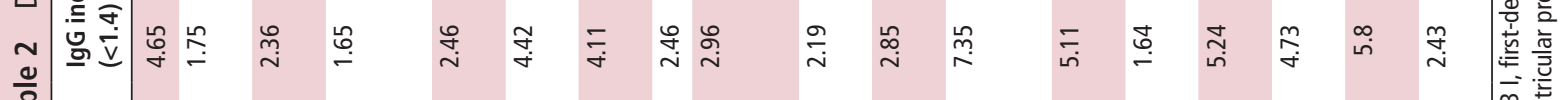

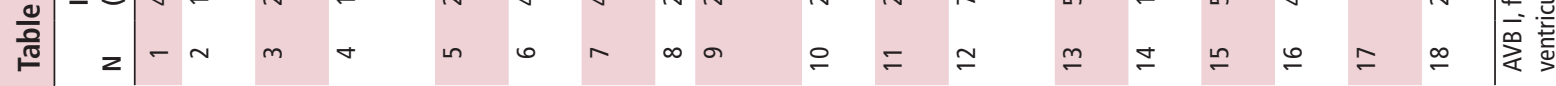




\begin{tabular}{|c|c|c|c|}
\hline & COVID-19+ & COVID-19- & $P$ value \\
\hline$n$ & 18 & 12 & \\
\hline Age (years) & $22(21-27)$ & $25(19.5-26.5)$ & 0.92 \\
\hline \multicolumn{4}{|l|}{ Spirometry } \\
\hline FVC (L) & $5.68(5.06-6.21)$ & $5.62(4.80-5.83)$ & 0.31 \\
\hline$\%$ of the theor & $102.5(92-112)$ & $103.5(95-105)$ & 0.69 \\
\hline $\mathrm{FEV}_{1}(\mathrm{~L})$ & $4.62(4.02-4.77)$ & $4.4(3.87-5.11)$ & 0.95 \\
\hline$\%$ of the theor & $96.5(91-104)$ & $102.5(94.5-108.5)$ & 0.39 \\
\hline $\operatorname{PEF}(\mathrm{L})$ & 9.25 (8.7-10.45) & $9.38(8.34-10.39)$ & 0.85 \\
\hline$\%$ of the theor & $89.5(84-100)$ & 89 (82.5-99.5) & 0.92 \\
\hline MVV (L/min) & $161.7(139.7-167)$ & $154(135.3-178.9)$ & 0.98 \\
\hline$\%$ of the theor & $98(91-104)$ & $99.5(90.5-110)$ & 0.72 \\
\hline \multicolumn{4}{|l|}{ Stress-test ECG } \\
\hline Resting HR (bpm) & $61.5(54-69)$ & $60.5(55-62.5)$ & 0.33 \\
\hline Max HR (bpm) & 180 (172-185) & $178(170-181.5)$ & 0.44 \\
\hline$\%$ of the theor & 91 (89-92) & 90.5 (87.5-92) & 0.63 \\
\hline Resting SBP (mm Hg) & $102.5(100-110)$ & $110(100-120)$ & 0.22 \\
\hline Resting DBP (mm Hg) & $62.5(60-70)$ & $62.5(60-72.5)$ & 0.55 \\
\hline Max SBP (mm Hg) & $190(170-200)$ & $190(175-195)$ & 1,00 \\
\hline $\operatorname{Max}$ DBP $(\mathrm{mm} \mathrm{Hg})$ & $77.5(70-85)$ & $75(70-80)$ & 0.63 \\
\hline \multicolumn{4}{|l|}{ Echocardiogram } \\
\hline LV EDD (mm) & $55.5(51.5-58)$ & $53.5(52.5-55.5)$ & 0.39 \\
\hline LV ESD (mm) & $33(31-37)$ & $33(32.5-34.5)$ & 0.85 \\
\hline IVSd (mm) & $10(9.5-10)$ & $10(9-10.25)$ & 0.92 \\
\hline PWd (mm) & $10(9.5-10)$ & $10(9-10.25)$ & 0.78 \\
\hline LV EDV (mL) & $153(126-168)$ & $137(131.5-153)$ & 0.25 \\
\hline LV EDV ind $\left(\mathrm{mL} / \mathrm{m}^{2}\right)$ & $73.5(63.1-85.3)$ & $71(63.9-75.2)$ & 0.35 \\
\hline LV ESV (mL) & $43.5(37-60)$ & $45(43.5-48.5)$ & 0.82 \\
\hline LV ESV ind $\left(\mathrm{mL} / \mathrm{m}^{2}\right)$ & $22.2(18.8-27.7)$ & $23.5(20.7-24.7)$ & 0.78 \\
\hline FS \% & $37(35-41)$ & $38(36.5-41)$ & 0.55 \\
\hline $\mathrm{EF} \%$ & $61(58-63)$ & $60.5(56.5-62.5)$ & 0.76 \\
\hline Mitral E:A ratio & $1.71(1.45-1.88)$ & $1.76(1.58-2)$ & 0.47 \\
\hline RVD1 (mm) & $44(41-45)$ & $43(39.5-45.5)$ & 0.69 \\
\hline RVD2 (mm) & $32.5(30-34)$ & $31(29-35.5)$ & 0.79 \\
\hline TAPSE (mm) & $19.5(19-22)$ & 20.5 (19-21) & 0.69 \\
\hline
\end{tabular}

Data are presented as median (IQR).

$B P M$, beats per minute; $D B P$, diastolic blood pressure; $L V E D D$, left ventricle enddiastolic diameter; LV EDV, left ventricle end-diastolic volume; EF, ejection fraction; LV ESD, left ventricle end-systolic diameter; LV ESV, left ventricle end-systolic volume; $\mathrm{FEV}_{1}$, forced expiratory volume in the first second; $\mathrm{FS}$, fractional shortening; FVC, forced vital capacity; HR, heart rate; ind, indexed for body surface area; IVSd, end-diastolic interventricular septum thickness; MVV, maximal voluntary ventilation; PEF, peak expiratory flow volume; PWd, end-diastolic posterior wall thickness; RVD1, right ventricle end-diastolic diameter at the base (4ch); RVD2, right ventricle end-diastolic mid-cavitary diameter (4ch); SBP, systolic blood pressure; TAPSE, tricuspid annular plane systolic excursion; Theor, theoretical for the age and body size.

between the pre-COVID-19 and the post-COVID-19 values with the Wilcoxon signed-rank test. The association between categorical variables was assessed by $\chi^{2}$ test. $\mathrm{P}<0.05$ was considered statistically significant.

All statistical analyses were performed with SPSS V.20.0 software.

\section{RESULTS}

Study population and clinical profiles

Thirty professional soccer players were included in the study. None of them had known cardiovascular or pulmonary disease. None of them had positive PCR swab and/or IgM class antibodies. Eighteen (60\%) players had negative swab and negative
IgM class but positive IgG class antibodies, so they were included in the COVID-19+ group. They were all asymptomatic for COVID-19-related symptoms at the time of the screening. Six (33.3\%) had been fully asymptomatic and found to be positive for SARS-CoV-2. Twelve (66.7\%) had complained of COVID19-related symptoms in the previous 3 months, but all resolved at least 15 days before the examination. The referred symptoms were fever $>37.5^{\circ} \mathrm{C}(\mathrm{n}=7,38.9 \%)$, ageusia $(\mathrm{n}=5,27.8 \%)$, asthenia $(n=5,27.8 \%)$, anosmia $(n=3,16.7 \%)$, cough $(n=2$, $11.1 \%)$, arthromyalgia $(n=2,11.1 \%)$, sore throat $(n=1,5.6 \%)$, headache $(n=1,5.6 \%)$ and nocturnal dyspnoea $(n=1,5.6 \%)$. None of the players complained of cardiovascular symptoms, either pre or post the presumed SARS-CoV-2 infection.

A detailed description of symptoms, data derived from the blood tests and instrumental findings among COVID-19+ players are reported in tables 1 and 2, including a comparison with the same parameters during the previous sports medical examination, about 1 year earlier.

Twelve (40\%) athletes had negative PCR swab and negative $\operatorname{IgM}$ and $\operatorname{IgG}$ class antibodies and were included in the COVID-19 - group. None of them complained of potentially COVID-19-related symptoms in the last 3 months and/or of cardiovascular symptoms. A comparison between COVID-19+ and COVID-19 - players with regard to spirometry, stress-test ECG and ECHO is shown in table 3.

A comparison of the same parameters between those dating back to the preseason of the previous year (on average 10 months before, range 9-11), available in 24 out of 30 players (15 COVID-19+ and 9 COVID-19-), and those following the outbreak is shown in table 4.

\section{Laboratory and instrumental findings in COVID-19+ group} before and after COVID-19 infection

In 18 COVID-19+ players, the vast majority of blood parameters after the infection remained in the normal ranges, with the exception of a slight increase in AST in 7 (38.9\%) cases, 4 of whom had increased values also at the previous control, a slight increase in creatinine in $4(22.2 \%), 2$ with increased values also at the previous control, an increase in IL-6 in 2 (11.1\%) and an increase in $\mathrm{TnI}$ in $1(5.6 \%)$ case. Furthermore, there was no statistically significant difference in the percentage of players in this group with increased AST and creatinine levels before and after the COVID-19 infection (respectively, 38.9\% vs $37.5 \%$, $\mathrm{p}=0.93$ and $12.5 \%$ vs $22.2 \%, \mathrm{p}=0.46$ ).

At the spirometry performed after the infection, a mild reduction in PEF was seen in 2 cases out of 18 (11.1\%), in one already present previously.

Resting ECG was normal (substantially the same) before and after the infection in all players, with some showing changes compatible with the 'athlete's heart', such as sinus bradycardia, first-degree atrioventricular block or incomplete right bundle branch block. ${ }^{10}$

Stress-test ECG showed rare, isolated ventricular premature beats (VPB) in one case (5.6\%) and rare, isolated supraventricular premature beats (SVPB) in one case $(5.6 \%)$ not present before. One (5.6\%) player had occasional SVPB already present previously. Finally, one (5.6\%) athlete had rare, isolated VPB and non-significant ST depression, but with no previous data we do not know if these were present before infection.

Oxygen saturation monitoring was normal at rest, on effort and on recovery in all players.

ECHO was normal (substantially the same) in all players before and after the infection. 


\begin{tabular}{|c|c|c|c|c|c|c|c|}
\hline & COVID-19+ pre & COVID-19+ post & $P$ value & & COVID-19-pre & COVID-19- post & P value \\
\hline $\mathrm{n}$ & 15 & 15 & & $n$ & 9 & 9 & \\
\hline Spirometry & & & & Spirometry & & & \\
\hline FVC (L) & $5.64(5.44-6.15)$ & $5.35(5.01-6.20)$ & $<0.05^{*}$ & $\mathrm{FVC}(\mathrm{L})$ & $5.76(5.24-5.86)$ & $5.66(5.03-5.8)$ & 0.95 \\
\hline$\%$ of the theor & $101(98-107.5)$ & $96(92-106.5)$ & 0.18 & $\%$ of the theor & $103(90-111)$ & $104(97-105)$ & 0.33 \\
\hline $\mathrm{FEV}_{1}(\mathrm{~L})$ & $4.91(4.77-5.02)$ & $4.62(4.08-4.86)$ & $<0.05^{*}$ & $\mathrm{FEV}_{1}(\mathrm{~L})$ & $4.77(4.5-5.22)$ & $4.52(4.17-5.03)$ & 0.09 \\
\hline$\%$ of the theor & $104(99-109)$ & $95(91.5-103)$ & $<0.05^{*}$ & $\%$ of the theor & $101(99-105)$ & $102(97-109)$ & 0.83 \\
\hline PEF (L) & $11.15(9.59-11.34)$ & $9.26(8.65-10.58)$ & $<0.05^{*}$ & PEF (L) & $11.2(8.9-11.8)$ & $9.52(8.85-10.36)$ & 0.05 \\
\hline$\%$ of the theor & $103(97.5-109.5)$ & $89(83-100.5)$ & $<0.05^{*}$ & $\%$ of the theor & $106(95-112)$ & $91(85-97)$ & 0.09 \\
\hline MVV (L/min) & 180.9 (151.6-197.9) & $161.7(141.3-170.2)$ & $<0.05^{*}$ & MVV (L/min) & $183.3(171.1-202.2)$ & $158.2(146-176.1)$ & 0.17 \\
\hline$\%$ of the theor & $110(90.5-122)$ & $97(91.5-104)$ & $<0.05^{*}$ & $\%$ of the theor & $116(99-119)$ & $100(95-109)$ & 0.17 \\
\hline Stress-test ECG & & & & Stress-test ECG & & & \\
\hline Resting HR (bpm) & $53(48-60)$ & $65(58-69)$ & $<0.05^{*}$ & Resting HR (bpm) & $60(57-62)$ & $61(55-62)$ & 0.86 \\
\hline Max HR (bpm) & $176(174-181)$ & $180(172-185)$ & 0.89 & Max HR (bpm) & 178 (177-182) & 179 (173-182) & 0.09 \\
\hline$\%$ of the theor & $90(88-92)$ & 91 (89-92) & 0.73 & $\%$ of the theor & $90(88-92)$ & 91 (89-92) & 0.26 \\
\hline Resting SBP (mm Hg) & $105(100-110)$ & $110(100-110)$ & 0.91 & Resting SBP (mm Hg) & $110(105-120)$ & $110(110-120)$ & 0.86 \\
\hline Resting DBP (mm Hg) & $65(65-70)$ & $65(60-70)$ & 0.53 & Resting DBP (mm Hg) & $70(60-70)$ & $65(60-70)$ & 0.67 \\
\hline $\operatorname{Max}$ SBP $(\mathrm{mm} \mathrm{Hg})$ & $190(185-200)$ & $190(180-205)$ & 0.72 & $\operatorname{Max}$ SBP (mm Hg) & $200(195-200)$ & 190 (180-195) & 0.23 \\
\hline $\operatorname{Max} \mathrm{DBP}(\mathrm{mm} \mathrm{Hg})$ & $75(65-80)$ & $75(70-80)$ & 0.26 & $\operatorname{Max} \mathrm{DBP}(\mathrm{mm} \mathrm{Hg})$ & $75(70-75)$ & $75(75-80)$ & 0.60 \\
\hline Echocardiogram & & & & Echocardiogram & & & \\
\hline LV EDD (mm) & $56(53.8-58)$ & $55(51.8-57.8)$ & 0.14 & $\operatorname{LV} \operatorname{EDD}(\mathrm{mm})$ & $53.3(52-54.5)$ & $53(52-55)$ & 0.49 \\
\hline $\operatorname{LV}$ ESD (mm) & $35(32-37.5)$ & $34(31-37.5)$ & 0.54 & $\operatorname{LV}$ ESD (mm) & $32.5(30-35)$ & $33(32-35)$ & 0.89 \\
\hline IVSd (mm) & $9.9(9.6-10)$ & $10(9.75-10)$ & 0.95 & IVSd (mm) & $9.63(9-10.3)$ & $10(9-10.5)$ & 0.40 \\
\hline $\mathrm{PWd}(\mathrm{mm})$ & $9.9(9.6-10)$ & $10(9.75-10)$ & 0.59 & $\mathrm{PWd}(\mathrm{mm})$ & $9.63(9-10)$ & $10(9-10.5)$ & 0.36 \\
\hline LV EDV (mL) & $153.7(139.7-166.6)$ & $151(126.5-168)$ & 0.47 & LV EDV (mL) & $136.5(130-144.4)$ & $134.5(131-146)$ & 0.84 \\
\hline LV EDV ind $\left(\mathrm{mL} / \mathrm{m}^{2}\right)$ & $75.5(67.5-82.9)$ & $73.5(61.6-84.9)$ & 0.41 & LV EDV ind $\left(\mathrm{mL} / \mathrm{m}^{2}\right)$ & $68.1(63.2-73.1)$ & $68.8(62.9-71.6)$ & 0.88 \\
\hline LV ESV (mL) & $50.9(40.9-59.9)$ & $46(37.5-62)$ & 0.89 & LV ESV (mL) & $42.5(35-51)$ & $45(42-45)$ & 0.96 \\
\hline LV ESV ind $\left(\mathrm{mL} / \mathrm{m}^{2}\right)$ & $26.1(19.8-28.7)$ & $23.6(18.3-30)$ & 0.78 & LV ESV ind $\left(\mathrm{mL} / \mathrm{m}^{2}\right)$ & $22.3(17.6-25.9)$ & $22.9(20-24.6)$ & 0.88 \\
\hline FS \% & $36(35-39.5)$ & $37(34.5-40)$ & 0.58 & FS $\%$ & $36.5(36-41)$ & $37(36-40)$ & 0.83 \\
\hline $\mathrm{EF} \%$ & $57(57-62.5)$ & $60(58-63)$ & 0.81 & $\mathrm{EF} \%$ & $58(56-60)$ & $62(56-63)$ & 0.24 \\
\hline Mitral E:A ratio & $1.87(1.63-2)$ & $1.6(1.45-1.86)$ & 0.21 & Mitral E:A ratio & $1.88(1.64-2.25)$ & $1.74(1.5-2)$ & 0.24 \\
\hline
\end{tabular}

Data are presented as median (IQR).

*statistically significant difference.

BPM, beats per minute; DBP, diastolic blood pressure; LV EDD, left ventricle end-diastolic diameter; LV EDV, left ventricle end-diastolic volume; EF, ejection fraction; LV ESD, left ventricle end-systolic diameter; LV ESV, left ventricle end-systolic volume; FEV ${ }_{1}$, forced expiratory volume in the first second; FS, fractional shortening; FVC, forced vital capacity; $\mathrm{HR}$, heart rate; ind, indexed for body surface area; IVSd, end-diastolic interventricular septum thickness; MVV, maximal voluntary ventilation; PEF, peak expiratory flow volume; PWd, end-diastolic posterior wall thickness; SBP, systolic blood pressure; Theor, theoretical for the age and body size.

Holter monitoring, including a training session, showed rare or occasional, isolated SVPB and/or VBP in all athletes. We did not have previous Holter data, so we cannot know if these arrhythmias were present before the infection. However, no players had frequent and/ or complex arrhythmias of clinical relevance. ${ }^{11-13}$

The post-COVID-19 chest CT was normal in all athletes.

A contrast-enhanced cardiac magnetic resonance (CMR) was performed only in one player who had normal resting and stress-test ECG, ECHO, Holter and chest CT (figure 1A-D) but increased level of TnI. CMR was completely normal (figure 1E-M).

Comparison between COVID-19+ and COVID-19- players Considering data from spirometry, stress-test ECG and ECHO performed at post-COVID-19 screening, no significant differences were found between COVID-19+ and COVID-19groups (table 3 ).

\section{Comparison between pre-COVID-19 and post-COVID-19} parameters in COVID-19+ and COVID-19- players

When comparing the same parameters before and after the COVID-19 outbreak (table 4), players in the COVID-19+ group showed a statistically significant reduction in almost all spirometry parameters after infection. A slight lowering (not significant) of these parameters was also observed in the COVID-19- group.

As regards data derived from stress-test ECG and ECHO, no statistically significant differences were found between the pre-COVID-19 and the post-COVID-19 values in both groups, with the exception of higher post-COVID-19 resting HR in the COVID-19+ group.

\section{DISCUSSION}

Our study highlights important data to inform the resumption of training and competitions without health risks in athletes who had asymptomatic or mildly symptomatic SARS-CoV-2 infection. In the soccer team we examined, serological tests revealed that 6 out of $30(20 \%)$ players had contracted SARS-CoV-2 in an asymptomatic manner.

In our cohort, none of the COVID-19+ soccer players showed signs of cardiovascular impairment after clinical resolution. Even in the player showing increased TnI level, which could be suspicious for myocarditis or 'myocardial damage', no anomalies were found on resting and stress-test ECG, Holter, ECHO, chest CT and CMR (figure 1). In this case, it is difficult to explain the 

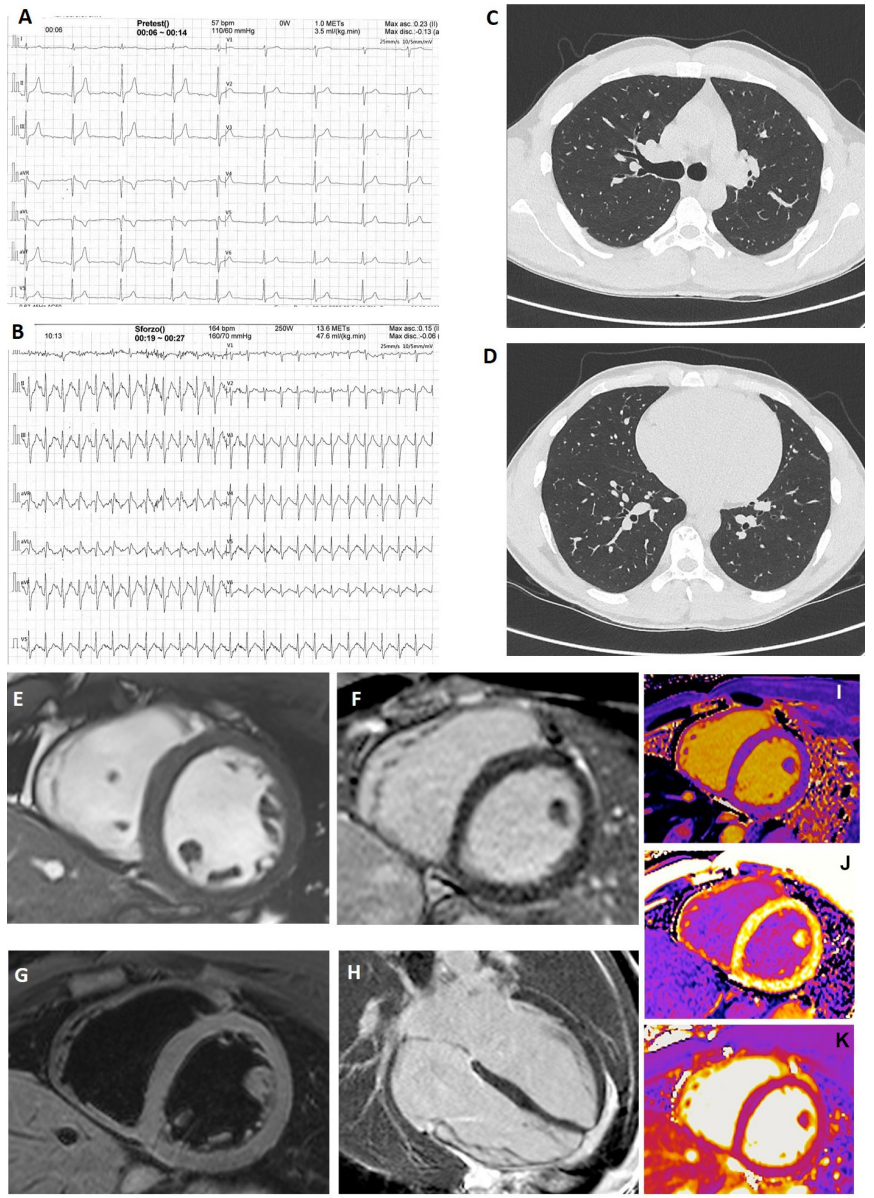

Figure 1 Instrumental findings in a player with increased troponin I level. In the only SARS-CoV-2-positive player (asymptomatic) with increased troponin I level, resting (A) and stress-test (B) ECG were normal. Chest CT at the level of the plane passing through the upper right lobar bronchus (C) and of the plane passing through lung bases (D) was absolutely normal. (E-M) Cardiac magnetic resonance images acquired using a 1.5 T Siemens Aera (Siemens Healthcare, Erlangen, Germany). (E) Short-axis cine balanced steady-state free precession showed normal left ventricle end-diastolic volume, wall thickness and motion. (F) Short-axis T2 image showed no oedema. (G, H) Short-axis and four-chamber views showed no alteration of late gadolinium enhancement. (I, J) Short-axis T1 native and T1 postcontrast maps showed normal values of T1 and extracellular volume. (K) T2 map showed no oedema.

TnI elevation because we do not have previous TnI values for comparison. Microscopic myocardial damage is conceivable on a microvascular basis, ${ }^{14} 15$ although this may not be significant as to give visible signs even on CMR. We planned to keep the player at rest and repeat the dosage of TnI every 7 days until normalisation before resuming normal training. The player's TnI progressively decreased and normalised after 4 weeks, so we allowed resumption of training with careful clinical monitoring. Similar considerations can be done for players who showed elevated levels in IL-6 with normal CRP levels but without signs of cardiovascular damage, attributable to a residual inflammatory state or possibly to a psychophysical state of stress. ${ }^{16}{ }^{17} \mathrm{In}$ every case, in our opinion, such anomalies do not represent a contraindication to resumption of training if the other investigations are normal. Similarly, the slight increase in AST and creatinine values found in some players at post-COVID-19 screening has no clinical significance given the high frequency of these occurrences in athletes. ${ }^{18} 19$

Our results also suggest that additional blood tests such as D-dimer or coagulation tests are unnecessary given that clinically relevant thromboembolic disease in a healed and asymptomatic athlete is unlikely.

Furthermore, no relevant differences were found in the comparison of ECG and ECHO in COVID-19+ versus COVID19- soccer players, as well as in the comparison of the same parameters in the two groups before and after the outbreak.

The Holter, inclusive of a heavy training session, performed only in COVID-19+ players, showed rare or occasional, isolated SVPB and VBP, a common finding in healthy athletes. ${ }^{13}$ We do not know if these were present earlier, but in no case were the arrhythmias a cause for concern. ${ }^{11-13}$ Given this, Holter monitoring does not seem necessary as a routine test in asymptomatic or mildly symptomatic SARS-CoV-2-positive athletes, if resting and stress-test ECGs do not show abnormalities.

The only parameters that appeared significantly reduced in COVID-19+ players were in spirometry. In every case, they remained in the normal range in all subjects, except for two with a mild reduction in PEF (one with similar values before). In our opinion, given the absolute normality of chest CT and of oxygen saturation even at maximal stress-test ECG, this can be attributable to the detraining period of 2 months or more due to the pandemic, rather than to COVID-19-related pulmonary damage. The players who suffered mild symptoms probably completely interrupted their home-based training programmes for 1 or more weeks, a distinction from the COVID-19players. This hypothesis is supported by the higher resting HR observed in COVID-19+ players post-COVID-19, suggesting deconditioning compared with pre-COVID-19 measurements. To verify this, we planned a spirometry retest after 1 month of retraining at full volumes. At this second control, almost all values in the COVID-19+ group improved. The only parameter that still showed a statistically significant reduction $(\mathrm{p}<0.05)$ compared with the pre-COVID-19 values was absolute FEV (while the percentage of the theoretical one had $\mathrm{p}>0.05$ ).

\section{Study limitations}

The main study limitation is the relatively small number of athletes investigated. While the study size limits definitive conclusions, this is the first study to provide any data on the comprehensive cardiovascular evaluation of athletes with prior asymptomatic or mild SARS-CoV-2 infection, with a comparison with COVID19 - athletes and data from previous years. Given the urgent need for information regarding safe resumption of sports, and that it is difficult to find a sufficient number of top athletes thoroughly screened after mild or asymptomatic SARS-CoV-2 infection, we believe this work will be of value to sports physicians and cardiologists. Other limitations are the specific cohort of athletes analysed (men, professional soccer players) in one single centre, the absence of a longitudinal follow-up and the CMR performed only in one player.

\section{CONCLUSIONS}

Our study confirms that screening athletes by personal history, PCR swabs and anti-SARS-CoV-2 antibodies before restarting training and competitions will provide evidence of previous SARS-CoV-2 infection where additional cardiac evaluation may be warranted. None of the players we examined extensively revealed adverse outcomes after clinical recovery, indicating that an extensive cardiorespiratory and haematological 
screening is 'excessive' in most cases, and routine standards for preparticipation cardiovascular screening as guided by the requirements of the sports league may be sufficient. Of course, further investigations are not needed in the SARS-CoV-2negative athletes, especially if totally asymptomatic during the pandemic period.

A protocol like the one we performed, inclusive of spirometry, resting ECG, stress-test ECG with oxygen saturation monitoring and ECHO, is ethically reasonable in SARS-CoV-2-positive athletes from a preventive perspective, although the probability that it will find relevant cardiopulmonary anomalies is low.

A different and more cautious approach seems reasonable in the presence of increased $\mathrm{TnI}$ values and/or signs of myocardial disfunction/damage on ECHO or stress-test ECG. In these cases, a contrast-enhanced CMR is recommended. Even if all the investigations are normal, we recommend waiting until the TnI levels return to normal before resumption of training, together with close clinical follow-up.

Finally, chest CT is absolutely not necessary as a screening tool, even in the presence of mild reduction in spirometry parameters. It should be reserved and preferably preceded by cardiopulmonary exercise testing in athletes who show symptoms such as dyspnoea on effort and/or significant lowering of spirometry values and/or relevant anomalies of oxygen saturation on effort.

\section{What are the findings?}

- In professional soccer players who have had asymptomatic mild SARS-CoV-2 infection, an extensive cardiorespiratory and haematological screening, including targeted blood tests, spirometry, resting ECG, stress-test ECG with oxygen saturation monitoring and echocardiogram, did not identify adverse medical conditions that would limit sports participation.

\section{How might it impact on clinical practice in the future?}

- While ethically reasonable, a comprehensive evaluation before resuming training and competitions is excessive in most athletes with asymptomatic/mild SARS-CoV-2 infection, with low probability of finding relevant anomalies.

Contributors All authors gave substantial contribution to the conception or design of the work, or the acquisition, analysis or interpretation of data. All authors gave substantial contribution to drafting the work or revising it critically for important intellectual content. All authors approved the version published. All authors agreed to be accountable for all aspects of the work in ensuring that questions related to the accuracy or integrity of any part of the work are appropriately investigated and resolved.

Funding The authors have not declared a specific grant for this research from any funding agency in the public, commercial or not-for-profit sectors.

Competing interests None declared.

Patient and public involvement Patients and/or the public were not involved in the design, or conduct, or reporting, or dissemination plans of this research.

Patient consent for publication Not required.
Ethics approval The whole study was conducted according to the GCP and the Helsinki Declaration. The study design was approved by the Ethics Committee of the Policlinico Universitario 'A. Gemelli' Foundation of Rome (protocol ID 3246).

Provenance and peer review Not commissioned; externally peer reviewed.

Data availability statement Data are available upon reasonable request. All data relevant to the study are included in the article or uploaded as supplementary information. Deidentified participant data are available in a database held by the Sports Medicine Unit of the University Hospital Policlinico A Gemelli. They are not available for reuse. Additional information can be requested by contacting the corresponding author (gervasi.salvatore.md@gmail.com).

Open access This is an open access article distributed in accordance with the Creative Commons Attribution Non Commercial (CC BY-NC 4.0) license, which permits others to distribute, remix, adapt, build upon this work non-commercially, and license their derivative works on different terms, provided the original work is properly cited, appropriate credit is given, any changes made indicated, and the use is non-commercial. See: http://creativecommons.org/licenses/by-nc/4.0/.

\section{ORCID iD}

Salvatore Francesco Gervasi http://orcid.org/0000-0002-8933-6955

\section{REFERENCES}

1 Phelan D, Kim JH, Chung EH. A game plan for the resumption of sport and exercise after coronavirus disease 2019 (COVID-19) infection. JAMA Cardiol 2020. doi:10.1001/jamacardio.2020.2136. [Epub ahead of print: 13 May 2020].

2 Baggish A, Drezner JA, Kim J, et al. Resurgence of sport in the wake of COVID-19: cardiac considerations in competitive athletes. Br J Sports Med 2020;54:1130-1.

3 Driggin E, Madhavan MV, Bikdeli B, et al. Cardiovascular considerations for patients, health care workers, and health systems during the COVID-19 pandemic. J Am Coll Cardiol 2020;75:2352-71

4 Long B, Brady WJ, Koyfman A, et al. Cardiovascular complications in COVID-19. Am J Emerg Med 2020;38:1504-7.

5 Vessella T, Zorzi A, Merlo L, et al. The Italian preparticipation evaluation programme: diagnostic yield, rate of disqualification and cost analysis. Br I Sports Med 2020;54:231-7.

6 Narducci ML, Pelargonio G, La Rosa G, et al. Role of extensive diagnostic workup in young athletes and nonathletes with complex ventricular arrhythmias. Heart Rhythm 2020;17:230-7.

7 Gervasi SF, Palumbo L, Cammarano M, et al. Coronary atherosclerosis in apparently healthy master athletes discovered during pre-PARTECIPATION screening. Role of coronary CT angiography (CCTA). Int I Cardiol 2019;282:99-107.

8 Palmieri V, Gervasi S, Bianco M, et al. Anomalous origin of coronary arteries from the "wrong" sinus in athletes: Diagnosis and management strategies. Int I Cardiol 2018;252:13-20

9 Decree of the Italian Ministry of Health. [Rules on the health protection of professional sportsmen]. Gazzetta Ufficiale della Repubblica Italiana, 1995.

10 Sharma S, Drezner JA, Baggish A, et al. International recommendations for electrocardiographic interpretation in athletes. Eur Heart J 2018;39:1466-80.

11 authors V, Comitato C. Protocolli cardiologici per IL giudizio di idoneit allo sport agonistico 2017. Med Sport 2018;71:1-121.

12 Zipes DP, Link MS, Ackerman MJ, et al. Eligibility and Disqualification recommendations for competitive athletes with cardiovascular abnormalities: Task force 9: arrhythmias and conduction defects: a scientific statement from the American heart association and American College of cardiology. Circulation 2015;132:e315-25.

13 Biffi A, Delise P, Zeppilli P, et al. Italian cardiological guidelines for sports eligibility in athletes with heart disease: Part 1. J Cardiovasc Med 2013;14:477-99.

14 Babapoor-Farrokhran S, Gill D, Walker J, et al. Myocardial injury and COVID-19: possible mechanisms. Life Sci 2020;253:117723.

15 Peretto G, Sala S, Caforio ALP. Acute myocardial injury, MINOCA, or myocarditis? improving characterization of coronavirus-associated myocardial involvement. Eur Heart J 2020;41:2124-5.

16 Rohleder N, Aringer M, Boentert M. Role of interleukin-6 in stress, sleep, and fatigue. Ann N Y Acad Sci 2012;1261:88-96.

17 Bob P, Raboch J, Maes M, et al. Depression, traumatic stress and interleukin-6. J Affect Disord 2010;120:231-4.

18 Fallon KE. The clinical utility of screening of biochemical parameters in elite athletes: analysis of 100 cases. Br J Sports Med 2008;42:334-7.

19 Banfi G, Colombini A, Lombardi G, et al. Metabolic markers in sports medicine. Adv Clin Chem 2012:56:1-54 